\title{
ePlant in 2021: New Species, Viewers, Data Sets, and Widgets
}

\author{
Ben Waese-Perlman*1, Asher Pasha*1, Chantal Ho${ }^{1}$, Amirahmad Azhieh ${ }^{1}$, Yushan $\mathrm{Liu}^{1}$, Alexander \\ Sullivan ${ }^{1}$, Vincent Lau ${ }^{1}$, Eddi Esteban ${ }^{1}$, Jamie Waese ${ }^{1}$, George Ly ${ }^{1}$, Cornelia Hooper ${ }^{3}$, S. Evan Staton ${ }^{7}$, \\ Nicholas Brereton ${ }^{8}$, Cuong Le ${ }^{6}$, Rex Nelson ${ }^{9}$, Shelley Lumba ${ }^{1}$, David Goodstein ${ }^{2}$, A. Harvey Millar ${ }^{3}$, Isobel \\ Parkin $^{4}$, Lewis Lukens ${ }^{5}$, Juergen Ehlting ${ }^{6}$, Loren Rieseberg ${ }^{7}$, Frédéric Pitre ${ }^{8}$, Anne Brown ${ }^{9}$, Nicholas J. \\ Provart ${ }^{1 @}$ \\ 1. Department of Cell \& Systems Biology / Centre for the Analysis of Genome Evolution and Function, University of Toronto, CANADA \\ 2. Phytozome, Joint Genome Institute, Lawrence Berkeley National Laboratory, U.S.A. \\ 3. School of Molecular Sciences, ARC Centre for Plant Energy Biology - University of Western Australia, AUSTRALIA \\ 4. Agriculture and Agrifood Canada / University of Saskatchewan, CANADA \\ 5. Department of Plant Agriculture, University of Guelph, CANADA \\ 6. Department of Biology, University of Victoria, CANADA \\ 7. Department of Botany, University of British Columbia, CANADA \\ 8. Department of Biological Sciences, Université de Montréal, CANADA \\ 9. United States Department of Agriculture, U.S.A. \\ @ Correspondence: nicholas.provart@utoronto.ca \\ * Authors contributed equally to this work
}

\section{Abstract}

ePlant was introduced in $\mathbf{2 0 1 7}$ for exploring large Arabidopsis thaliana data sets from the kilometre to nanometre scales. In the past four years we have used the ePlant framework to develop ePlants for 15 agronomically-important species: maize, poplar, tomato, Camelina sativa, soybean, potato, barley, Medicago truncatula, eucalyptus, rice, willow, sunflower, Cannabis sativa, wheat and sugarcane. We also updated the interface to improve performance and accessibility, and added two new views to the Arabidopsis ePlant - the Navigator and Pathways viewers. The former shows phylogenetic relationships between homologs in other species and their expression pattern similarities, with links to view data for those genes in the respective ePlants. The latter shows Plant Reactome metabolic reactions. We also describe new Arabidopsis data sets including single cell RNA-seq data from roots, and how to embed ePlant eFP expression pictographs into any web page.

\section{Introduction}

Vast amounts of biological data have been generated over the past 15 years. Traditional methods of analyzing data in multiple formats can add a layer of friction to the creative processes of hypothesis generation. We introduced ePlant four years ago to help plant biologists explore and make connections between large Arabidopsis thaliana data sets for any given gene/gene product (Waese et al., 2017). It 
includes views for exploring expression differences in ecotypes at a kilometre scale, expression levels in tissues and organs at the centimetre scale, sub-cellular localization at the millimetre scale, protein-DNA and protein-protein interactions at the micrometre scale and, finally, the tertiary structure of the molecule itself at the nanometre scale. Combining multiple views into one visual analytic platform allows researchers to ask and answer complex biological questions about a gene of interest using a single, user-friendly interface.

Development of ePlant has continued since the original paper was published. Several new views have been added and the user interface has been updated to improve performance and accessibility. The new views include a Navigator viewer, a Pathways viewer, and seven new eFP viewers based on RNA-seq data. In addition, 15 new ePlants have been developed for agronomically-important species beyond Arabidopsis thaliana. Last, we have made it easy to embed ePlant eFP images showing expression patterns into any web page as a widget.

\section{Results}

\section{New ePlant Design with Improved Accessibility and Performance}

Since the original Waese et al. (2017) paper, ePlant interface has undergone a major update (see Figure 1). The colours have been updated to improve contrast, infrequently used features were removed to reduce clutter, and animated page transitions were removed to eliminate negative experiences that motion effects cause for some users. In addition, gene loading times have improved by up to $300 \%$ and several bugs have been fixed that used to cause unpredictable behavior when asynchronous events did not happen as expected.

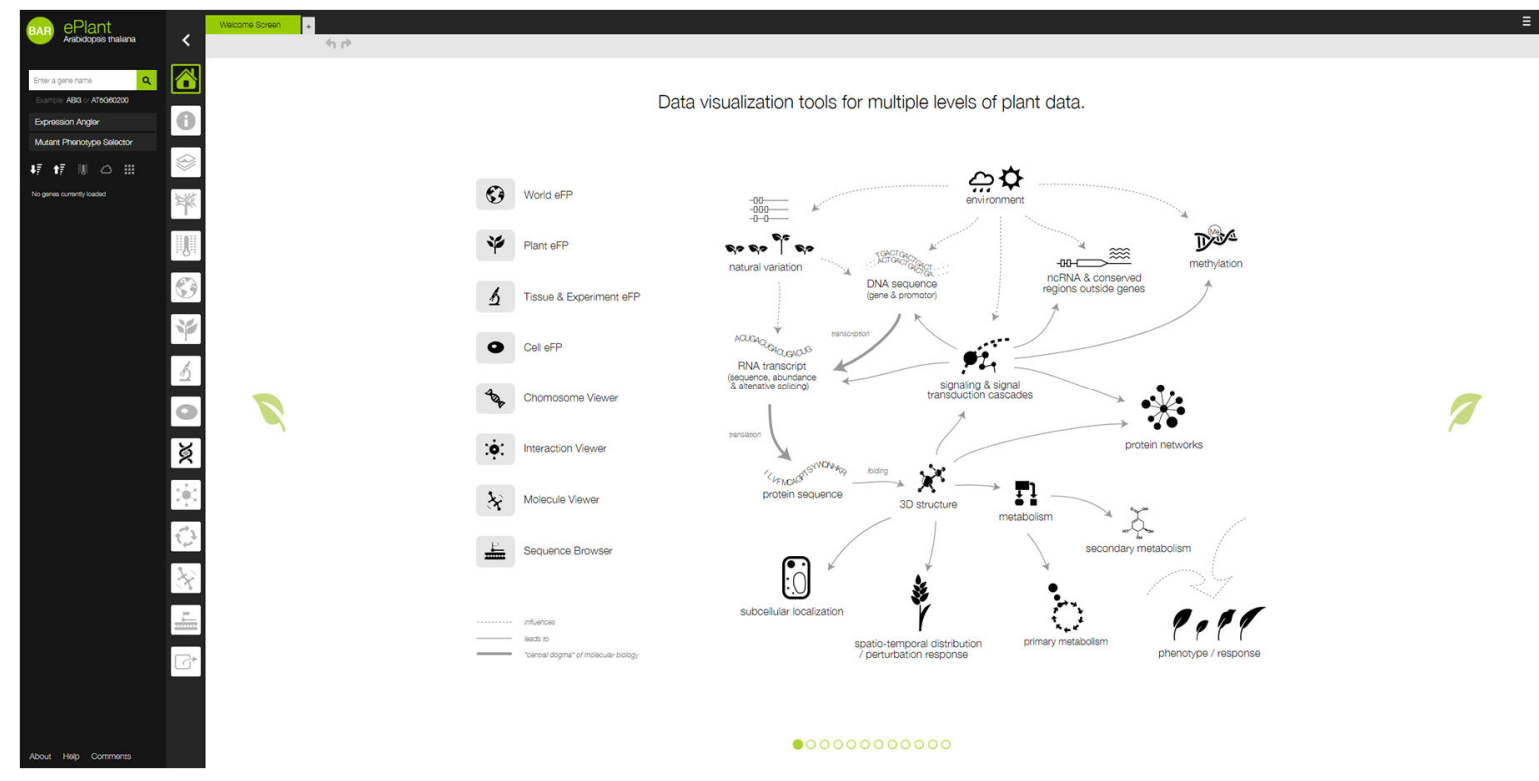

Figure 1: The new ePlant home page.

To improve contrast, we swapped the background colours for darker ones, removed gradients and changed text colours, making much of ePlant compatible with the higher WCAG "AAA" standard for 
enhanced contrast, which requires a ratio of at least $7: 1^{1}$. The original design did not meet the WCAG "AA" standard. The new design uses the colours shown in Figure 2.
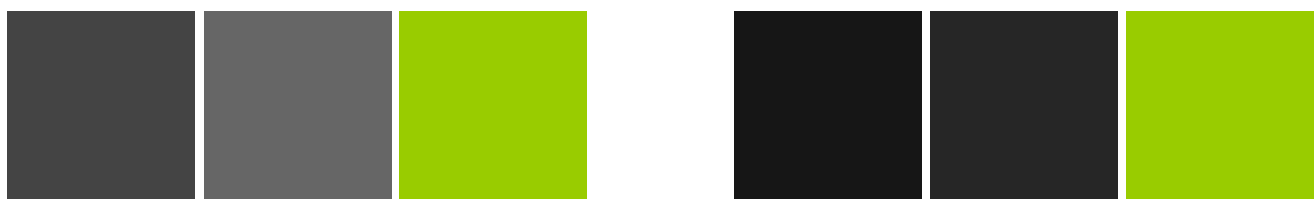

Figure 2: Original ePlant colours (left) \#444444, \#666666, \#99CC00; new colours (right) \#161616, \#262626, \#99CC00

We have maintained use of the "BAR green" as the highlight colour, despite darkening the background colours. By swapping the background colours to darker alternatives and removing the use of white text on a green background we were able to increase contrast significantly and create a more readable design (Figure 3). We also removed the animated transitions between views to address concerns from researchers with vestibular disorders².
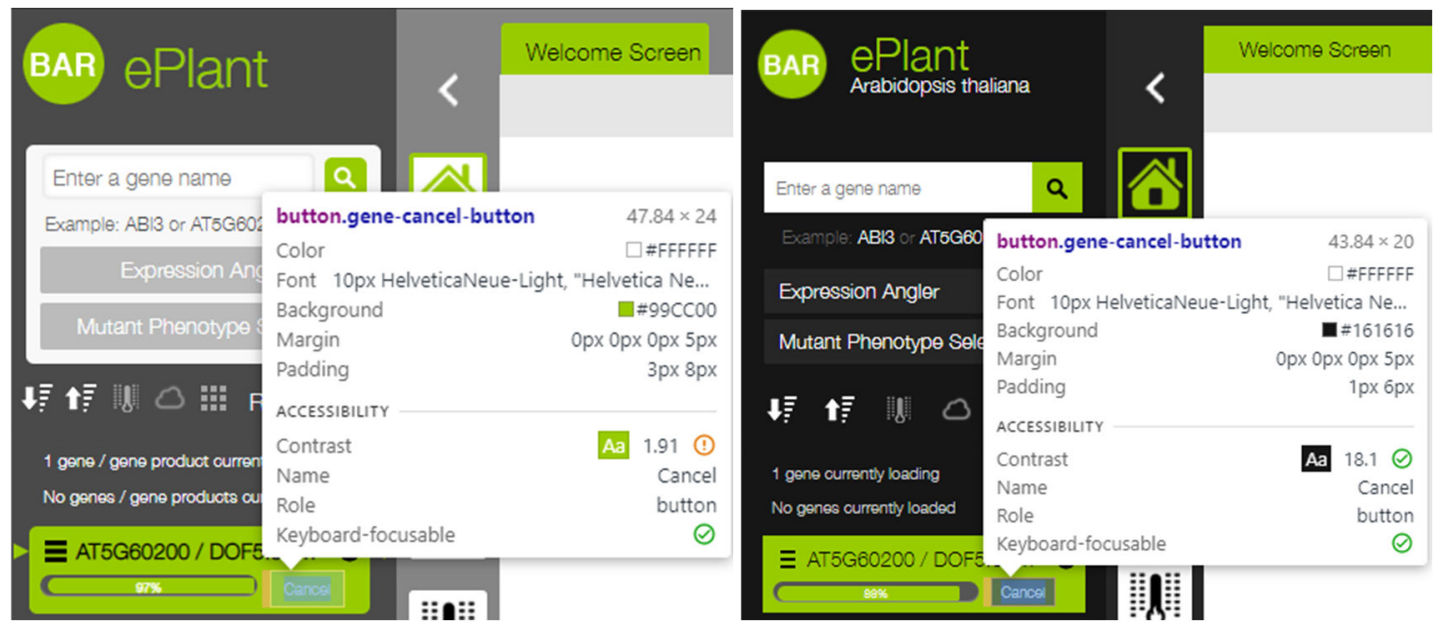

Figure 3: Contrast of the original ePlant design versus the new design. The original design (left) does not meet the WCAG "AA" level for contrast, while the new design (right) exceeds the "AAA" contrast level by a factor of more than two, as shown using Chrome's developer tools.

\section{A Small Farm of ePlants}

The original ePlant published by Waese et al. (2017) only supported data from Arabidopsis thaliana. We now have ePlants for another 15 species: Arabidopsis thaliana, maize, poplar, tomato, Camelina sativa, soybean, potato, barley, Medicago truncatula, eucalyptus, rice, willow, sunflower, Cannabis sativa, wheat and sugarcane (see Figure 4 and Table 1).

\footnotetext{
${ }^{1}$ https://www.w3.org/TR/WCAG21/\#contrast-minimum

2 https://alistapart.com/article/designing-safer-web-animation-for-motion-sensitivity/
} 


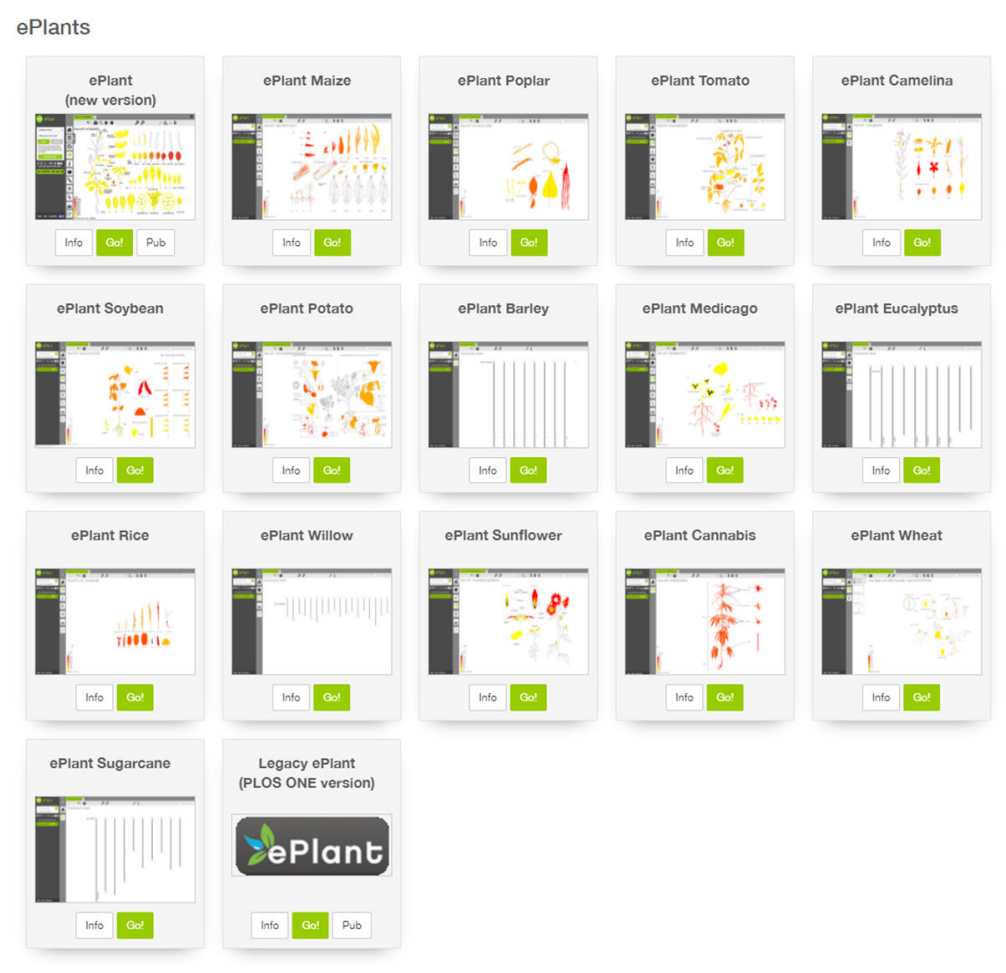

Figure 4: Accessing ePlants from the BAR homepage at http://bar.utoronto.ca.

Although they share a common interface, the ePlants are not all identical. The 16 ePlants draw data from a variety of databases, and different species have different views based on data availability, as shown in Table 1. The Arabidopsis ePlant continues to have the greatest number of views. We developed a pipeline for creating a new ePlant of interest and this is available at https://github.com/BioAnalyticResource/ePlant Pipeline. For several ePlants, we have predicted "structure-omes" using Phyre2 (Kelley et al., 2015). 
Table 1: Overview of Data Available in each ePlant. Icons are defined in Figure 1, with the exception of the first 4: the $\mathbf{0}$ icon denotes gene information, the icon denotes publications, the $=$ icon denotes ePlant Navigator, and the ill icon denotes the Heatmap Viewer. A new icon is the Pathways Viewer icon. ePlants are available at http://bar. utoronto.ca.

\begin{tabular}{|c|c|c|c|c|c|c|c|c|c|c|c|c|c|c|}
\hline ePlant $\backslash$ Viewer & ( & $\otimes$ & $\square$ & (1) & 3 & 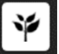 & 3 & - & 兑 & $\therefore:$ & 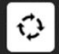 & $\neq$ & E & Data references \\
\hline Arabidopsis ePlant & - & - & $\bullet$ & - & • & - & • & - & - & • & - & - & - & As in Waese et al. (2017) and as discussed in this paper \\
\hline Maize ePlant & & - & & & & $\bullet$ & $\bullet$ & & $\bullet$ & $\bullet$ & & & - & $\begin{array}{l}\text { Downs et al. (2013), Opitz et al. (2014, 2016), (2016), Wang et } \\
\text { al. (2014), Li et al. (2010), Eveland et al. (2014) Hey et al. (2017), } \\
\text { Sekhon et al. (2011), Musungu et al. (2015), Goodstein et al. } \\
\text { (2011) }\end{array}$ \\
\hline Poplar ePlant & - & & & & $\bullet$ & $\bullet$ & $\bullet$ & & $\bullet$ & & & - & - & $\begin{array}{l}\text { Tuskan et al. (2006), Wilkins et al. (2009a, 2009b), Goodstein et } \\
\text { al. (2011) }\end{array}$ \\
\hline Tomato ePlant & - & & & & & $\bullet$ & $\bullet$ & $\bullet$ & $\bullet$ & & & & - & $\begin{array}{l}\text { Tomato Genome Consortium (2012), Koening et al. (2013), Toal } \\
\text { et al. (2018), Kajala et al. (in press), Chitwood et al. (2013), } \\
\text { Matas et al. (2011), Hooper et al. (2020), Goodstein et al. (2011) }\end{array}$ \\
\hline Camelina ePlant & & & & & & - & & & - & & & & & Kagale et al. (2016) \\
\hline Soybean ePlant & $\bullet$ & $\bullet$ & & & & $\bullet$ & $\bullet$ & & $\bullet$ & & & - & $\bullet$ & $\begin{array}{l}\text { Libault et al. (2010a, 2010b), Severin et al. (2010), Goodstein et } \\
\text { al. (2011) }\end{array}$ \\
\hline Potato ePlant & & & & & & - & $\bullet$ & & $\bullet$ & & & & $\bullet$ & $\begin{array}{l}\text { Massa et al. (2011), Potato Genome Sequecing Consortium } \\
\text { (2011), Goodstein et al. (2011) }\end{array}$ \\
\hline Barley ePlant & ○ & & & & & & $\bullet$ & & $\bullet$ & & & & & Thiel et al. (2021) \\
\hline Medicago ePlant & - & - & & & & - & $\bullet$ & & $\bullet$ & & & $\bullet$ & - & $\begin{array}{l}\text { Benedito et al. (2008), Righetti et al. (2015), Goodstein et al. } \\
\text { (2011) }\end{array}$ \\
\hline Eucalyptus ePlant & - & & & & & $\bullet$ & $\bullet$ & & $\bullet$ & & & & - & $\begin{array}{l}\text { Myburg et al. (2014), Bartholomé et al. (2015), Zhang et al. (in } \\
\text { prep.), Goodstein et al. (2011) }\end{array}$ \\
\hline Rice ePlant & & & & & & $\bullet$ & $\bullet$ & & $\bullet$ & - & & & - & $\begin{array}{l}\text { Jain et al. (2007), Wang et al. (2014), Li et al. (2007), Lasanthi- } \\
\text { Kudahettige(2007), Kajala et al. (in press), Ho et al. (2012), } \\
\text { Goodstein et al. (2011) }\end{array}$ \\
\hline Willow ePlant & $\bullet$ & & & & & & & & $\bullet$ & & & & $\bullet$ & Zhou et al. (2018), Goodstein et al. (2011) \\
\hline Sunflower ePlant & - & - & & & & - & & & - & & & & - & Badouin et al. (2017), Goodstein et al. (2011) \\
\hline Cannabis ePlant & & & & & & - & & & & & & & & Van Bakel et al. (2011) \\
\hline Wheat ePlant & & & & & & - & & & - & & & & & Ramírez-González et al. (2018) \\
\hline Sugarcane ePlant & & & & & & & $\bullet$ & & - & & & & & Hosaka et al. (submitted) \\
\hline
\end{tabular}




\section{Two New Viewers for the Arabidopsis ePlant}

\section{Navigator Viewer}

The Navigator Viewer as shown in Figure $\mathbf{5}$ provides a tree-based visualization of homologous genes in other species. It uses data from the BAR's Expressolog database (Patel et al., 2012) to construct a phylogenetic tree with leaf nodes representing the homologs from different species. For each gene, its species is reported along with the similarity at the sequence level and at the level of expression pattern similarity, based on the expressolog method developed by Patel et al. (2012). In this way, genes that are both sequence similar and have similar patterns of expression in equivalent tissues may be easily identified. Link-outs are also provided to each gene's corresponding ePlant and or eFP view. In addition, dynamic links to genomevolution.org's CoGe database (Lyons and Freeling, 2008) and to the Gramene database (Tello-Ruiz et al., 2018) are provided so that a researcher can easily access further information about syntenic relationships etc.

This tool was created with the goal of quickly and easily helping biologists find similar genes across the various ePlant species. If a biologist studying one species is familiar with a gene in another, this view allows them to make a connection between the two.

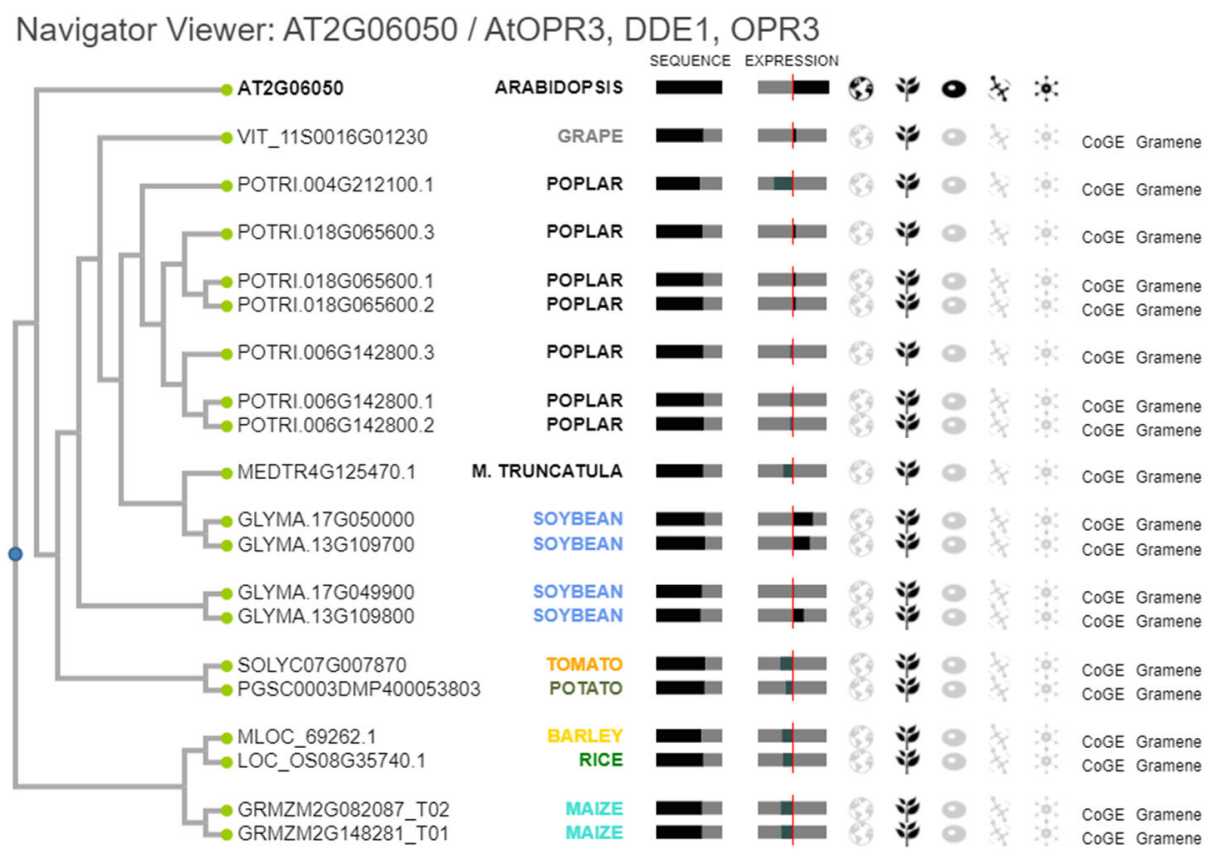

Figure 5: ePlant Navigator Viewer

\section{Pathways Viewer}

The Pathways Viewer shown in Figure 6 displays metabolic pathways associated with the active gene. It draws data from the Plant Reactome pathway database (Naithani et al., 2019) via application programming interface (API) calls and generates a pathway diagram. This is done using Cytoscape.js, a JavaScript-based graph theory library for network visualization and analysis (Franz et al., 2016). Nodes 
are laid out with a force directed layout known as COSE Bilkent ${ }^{3}$ and styled appropriately. All pathways available for a given gene are displayed as selectable tabs and a direct link to the pathway in the Plant Reactome pathway browser is provided for more information. In addition, hovering over the active gene label, which is denoted by bold text, calls up a tooltip which contains the Klepikova Plant eFP view for that gene, based on the Klepikova developmental atlas (Klepikova et al., 2016) - see the next section. The Klepikova eFP view can be used to assess in which parts of the plant the gene is strongly expressed, to help identify parts of the plant where the depicted reaction might be occurring.

The ePlant Pathways Viewer fills a gap in the conceptual hierarchy within ePlant, between the Interaction Viewer and the Molecule Viewer. Currently this view is only available for the Arabidopsis ePlant with data for over 236 pathways, 590 reactions and 1059 gene products and it is coming to the other ePlants soon. Plotting these pathways enables researchers to understand the biological context of the active gene product. The other views in ePlant show where in the cell the gene/macromolecule exists and how it is expressed, but this view is what makes it possible to answer the question "what is the gene doing in the cell" in a way that was not possible before.

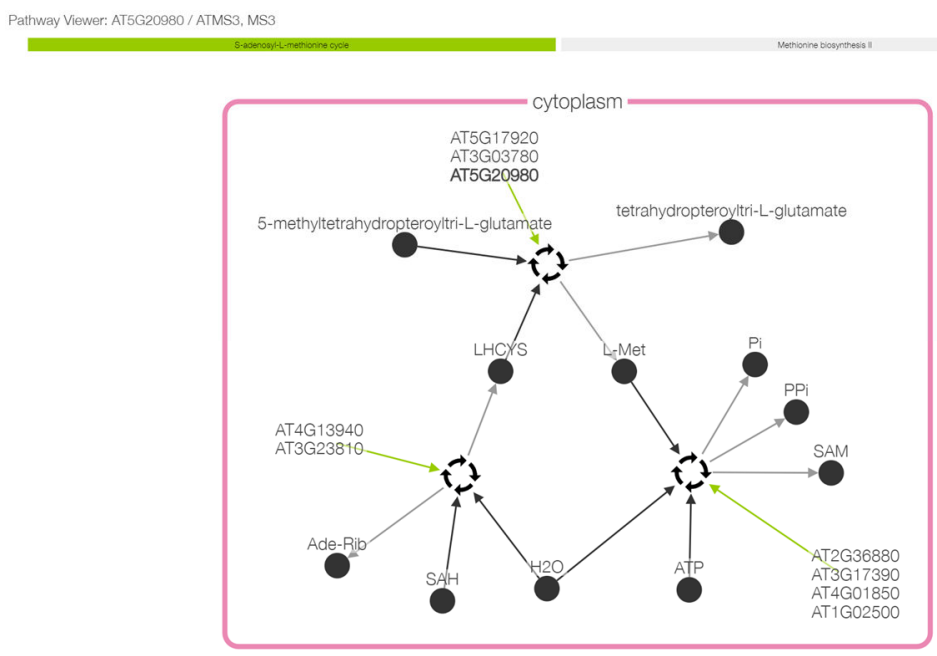

Figure 6: ePlant Pathways Viewer

\footnotetext{
${ }^{3}$ https://github.com/cytoscape/cytoscape.js-cose-bilkent
} 


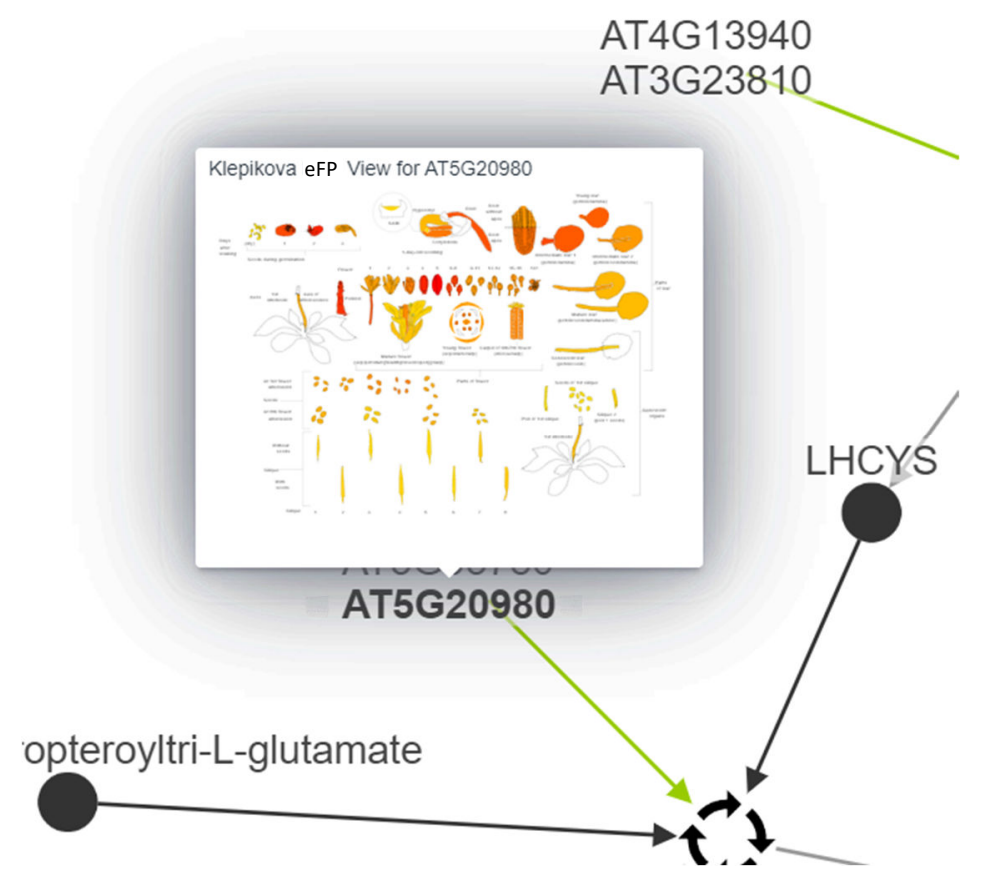

Figure 7: Klepikova Plant eFP tooltip in ePlant's Pathways Viewer, showing the expression pattern of At5g20980, ATMS3, involved in the S-adenosyl-L-methionine cycle, showing strong expression (red colour) in young leaves, flower buds, stems, and germinating seeds.

\section{New Data Sets}

The original ePlant publication introduced the Plant eFP Viewer and the Tissue and Experiment eFP Viewers with more than 20 different views. They display pictographs representing the level of expression of a queried gene in organs of the plant or in specific tissues or cell types, with intensity of expression represented by a colour gradient. Since the original publication in 2017, a number of new views have been added, with the Single Cell RNA Sequence eFP (Ryu et al., 2019) being one of six added in this new update, as shown in Figure 8. Most of the newly added data are based on RNA sequencing and include a new Plant eFP Viewer for the Klepikova RNA-seq-based developmental atlas shown in Figure 7 (Klepikova et al., 2016), a Germination view (Narsai et al., 2011), a Shoot Apex view (Tian et al., 2014), a Root Immunity Elicitation view (Rich-Griffin et al., 2020), and a Guard Cell Drought view (van Weringh et al., 2021).

We also updated the non-synonymous single nucleotide polymorphism data source for the Arabidopsis Molecule Viewer from the 1001 Proteomes site (Joshi et al., 2012), which had been deprecated, to the 1001 Genomes API (1001 Genomes Consortium, 2016). The advantage of doing this, apart from having a reliable data source, is that the 1001 Genomes API is more comprehensive, containing polymorphism data for almost twice as many Arabidopsis ecotypes. 


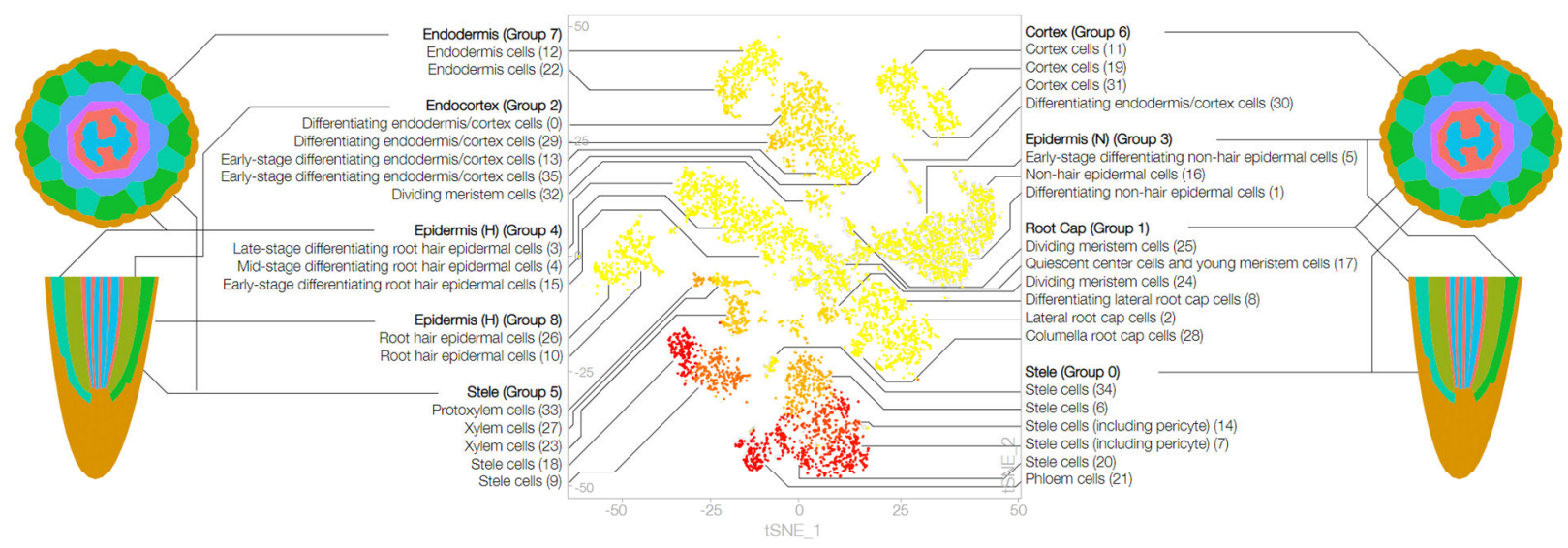

Figure 8: Single Cell RNA-seq eFP View for the gene At5g60200

\section{ePlant eFP Widgets}

We have developed SVG-based ePlant Plant eFP widgets for use in any website, as shown in Figure 9. See https://bar.utoronto.ca/ asullivan/ePlant Plant eFP/example/ for how to use these. The widgets behave similarly to the eFP views found within ePlant itself and are available for 88 different SVGs. They function after the same paradigm, with a gradient showing intensity of gene expression in different tissue regions or cell types taken from different parts of the plant.

\section{Discussion}

We present several useful updates to our ePlant tool. Improvements to the accessibility and performance of the interface makes for a better user experience. Fifteen new ePlants permit easy exploration of expression, interaction, subcellular localization and structure data across multiple agronomically-important species. We hope that the research communities for these species will be interested in adding data sets to these new ePlants in the future. Two new viewers, the Navigator and Pathways viewers, permit genes/gene products to be explored in the context of their homologs and biochemical pathways retrieved from Plant Reactome, respectively. A Plant eFP tooltip for the active gene/gene product in the latter viewer can help highlight in which organ a pathway might be operating. We have added new expression data sets based on RNA sequencing to the Tissue and Experiment Viewers, increasing the breadth of transcriptomic data viewable in a pictographic manner. Last, ePlant eFP widgets may be easily embedded in any webpage for any Arabidopsis gene with some straightforward code. 


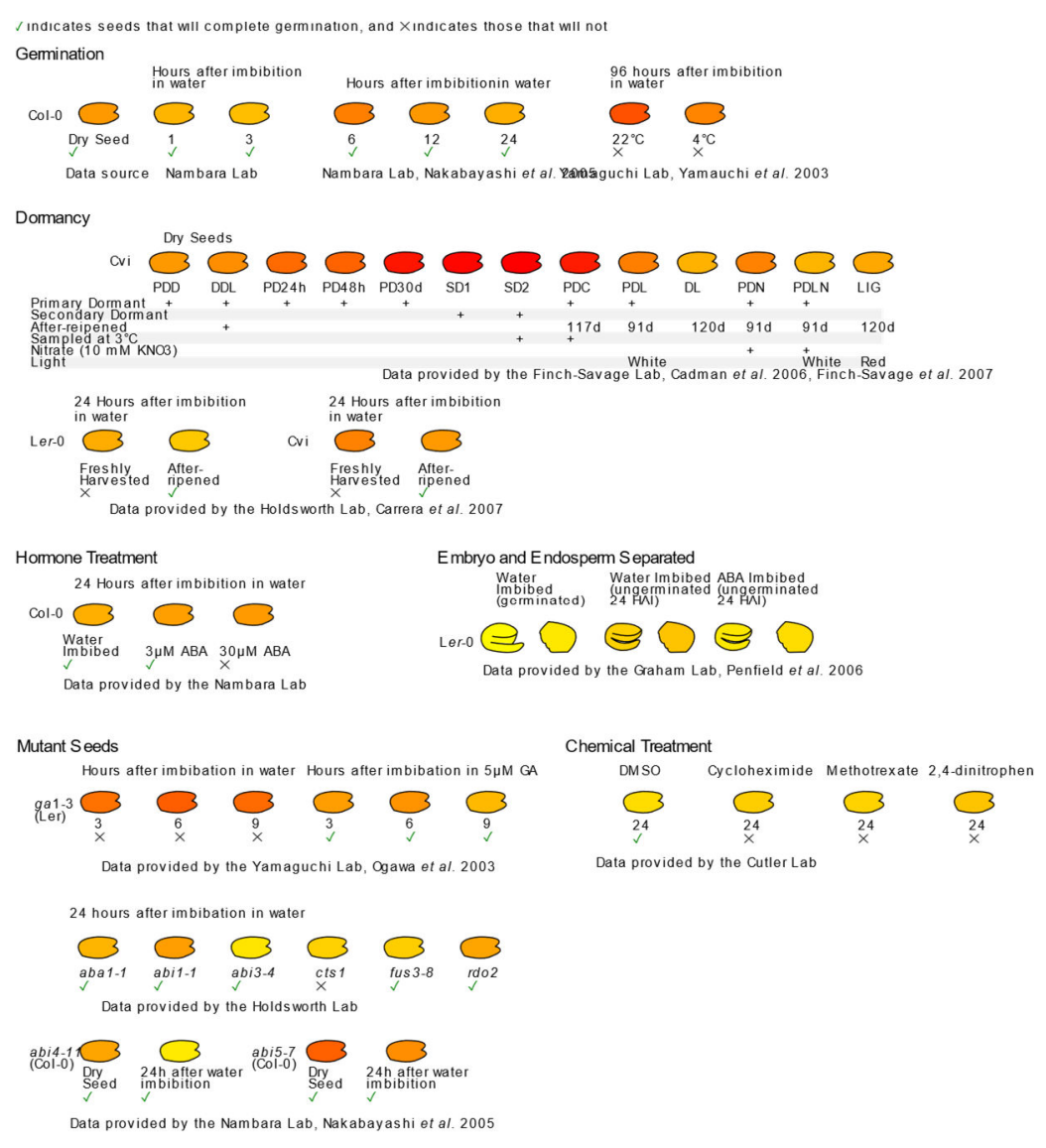

Figure 9: Seed ePlant eFP widget

\section{Acknowledgements, Contributions, and Funding}

We are grateful to Pankaj Jaiswal and Justin Preece from Oregon State University for helpful discussions about using the Plant Reactome API. BWP, AA and YL implemented the Pathways Viewer. BWP reskinned the $\mathrm{UI}$ and made many improvements to the ePlant code base. AP databased additional data not already on the BAR and customized ePlant code for new ePlants, and developed APIs for use in the Pathways Viewer. C. Ho developed the ePlant Navigator. AS created the ePlant Widgets. JW, VL and NJP supervised students ( $B W P, C H, Y L, A A)$, and assisted with image preparation for new ePlant views, as did EE. GL, SL, IP, LL, CL, RN, JE, LR, ES, FP and AB provided data, images, and feedback for new ePlants. AHM and C. Hooper provided data from CropPAL (http://crop-pal.org/). DG assisted with Phytozome JBrowse deployment in the ePlants. BWP, JW and NJP wrote and edited the manuscript and created the figures. NJP was funded by a grant from Genome Canada/Ontario Genomics (OG-128). 


\section{References}

1001 Genomes Consortium (2016). 1,135 Genomes Reveal the Global Pattern of Polymorphism in Arabidopsis thaliana. Cell 166: 481-491.

Badouin, H. et al. (2017). The sunflower genome provides insights into oil metabolism, flowering and Asterid evolution. Nature 546: 148-152.

Bartholomé, J., Mandrou, E., Mabiala, A., Jenkins, J., Nabihoudine, I., Klopp, C., Schmutz, J., Plomion, C., and Gion, J.-M. (2015). High-resolution genetic maps of Eucalyptus improve Eucalyptus grandis genome assembly. New Phytol. 206: 1283-1296.

Benedito, V.A. et al. (2008). A gene expression atlas of the model legume Medicago truncatula. Plant J. 55: 504-513.

Chitwood, D.H., Kumar, R., Headland, L.R., Ranjan, A., Covington, M.F., Ichihashi, Y., Fulop, D., Jiménez-Gómez, J.M., Peng, J., Maloof, J.N., and Sinha, N.R. (2013). A Quantitative Genetic Basis for Leaf Morphology in a Set of Precisely Defined Tomato Introgression Lines. Plant Cell 25: 2465-2481.

Downs, G.S., Bi, Y.-M., Colasanti, J., Wu, W., Chen, X., Zhu, T., Rothstein, S.J., and Lukens, L. (2013). A developmental transcriptional network for Zea mays defines coexpression modules. Plant Physiol.: pp.112.213231.

Eveland, A.L. et al. (2014). Regulatory modules controlling maize inflorescence architecture. Genome Res. 24: 431-443.

Franz, M., Lopes, C.T., Huck, G., Dong, Y., Sumer, O., and Bader, G.D. (2016). Cytoscape.js: a graph theory library for visualisation and analysis. Bioinformatics 32: 309-311.

Goodstein, D.M., Shu, S., Howson, R., Neupane, R., Hayes, R.D., Fazo, J., Mitros, T., Dirks, W., Hellsten, U., Putnam, N., and Rokhsar, D.S. (2011). Phytozome: a comparative platform for green plant genomics. Nucleic Acids Res. 40: D1178-D1186.

Hey, S., Baldauf, J., Opitz, N., Lithio, A., Pasha, A., Provart, N., Nettleton, D., and Hochholdinger, F. (2017). Complexity and specificity of the maize (Zea mays L.) root hair transcriptome. J. Exp. Bot. 68: $2175-2185$.

Ho, C.-L., Wu, Y., Shen, H.-B., Provart, N.J., and Geisler, M. (2012). A predicted protein interactome for rice. Rice N. Y. N 5: 15.

Hooper, C.M., Castleden, I.R., Aryamanesh, N., Black, K., Grasso, S.V., and Millar, A.H. (2020). CropPAL for discovering divergence in protein subcellular location in crops to support strategies for molecular crop breeding. Plant J. Cell Mol. Biol. 104: 812-827.

Jain, M., Nijhawan, A., Arora, R., Agarwal, P., Ray, S., Sharma, P., Kapoor, S., Tyagi, A.K., and Khurana, J.P. (2007). F-Box Proteins in Rice. Genome-Wide Analysis, Classification, Temporal and Spatial Gene Expression during Panicle and Seed Development, and Regulation by Light and Abiotic Stress. Plant Physiol. 143: 1467-1483. 
Joshi, H.J., Christiansen, K.M., Fitz, J., Cao, J., Lipzen, A., Martin, J., Smith-Moritz, A.M., Pennacchio, L.A., Schackwitz, W.S., Weigel, D., and Heazlewood, J.L. (2012). 1001 Proteomes: a functional proteomics portal for the analysis of Arabidopsis thaliana accessions. Bioinforma. Oxf. Engl. 28: 1303-1306.

Kagale, S., Nixon, J., Khedikar, Y., Pasha, A., Provart, N.J., Clarke, W.E., Bollina, V., Robinson, S.J., Coutu, C., and Hegedus, D.D. (2016). The developmental transcriptome atlas of the biofuel crop Camelina sativa. Plant J. 88: 879-894.

Kelley, L.A., Mezulis, S., Yates, C.M., Wass, M.N., and Sternberg, M.J.E. (2015). The Phyre2 web portal for protein modeling, prediction and analysis. Nat. Protoc. 10: 845-858.

Klepikova, A.V., Kasianov, A.S., Gerasimov, E.S., Logacheva, M.D., and Penin, A.A. (2016). A high resolution map of the Arabidopsis thaliana developmental transcriptome based on RNA-seq profiling. Plant J. 88: 1058-1070.

Koenig, D. et al. (2013). Comparative transcriptomics reveals patterns of selection in domesticated and wild tomato. Proc. Natl. Acad. Sci. 110: E2655-E2662.

Lasanthi-Kudahettige, R., Magneschi, L., Loreti, E., Gonzali, S., Licausi, F., Novi, G., Beretta, O., Vitulli, F., Alpi, A., and Perata, P. (2007). Transcript Profiling of the Anoxic Rice Coleoptile. Plant Physiol. 144: 218-231.

Li, M., Xu, W., Yang, W., Kong, Z., and Xue, Y. (2007). Genome-Wide Gene Expression Profiling Reveals Conserved and Novel Molecular Functions of the Stigma in Rice. Plant Physiol. 144: 1797-1812.

Li, P. et al. (2010). The developmental dynamics of the maize leaf transcriptome. Nat. Genet. 42: 10601067.

Libault, M., Farmer, A., Brechenmacher, L., Drnevich, J., Langley, R.J., Bilgin, D.D., Radwan, O., Neece, D.J., Clough, S.J., May, G.D., and Stacey, G. (2010a). Complete transcriptome of the soybean root hair cell, a single-cell model, and its alteration in response to Bradyrhizobium japonicum infection. Plant Physiol. 152: 541-552.

Libault, M., Farmer, A., Joshi, T., Takahashi, K., Langley, R.J., Franklin, L.D., He, J., Xu, D., May, G., and Stacey, G. (2010b). An integrated transcriptome atlas of the crop model Glycine max, and its use in comparative analyses in plants. Plant J. Cell Mol. Biol. 63: 86-99.

Lyons, E. and Freeling, M. (2008). How to usefully compare homologous plant genes and chromosomes as DNA sequences. Plant J. 53: 661-673.

Massa, A.N., Childs, K.L., Lin, H., Bryan, G.J., Giuliano, G., and Buell, C.R. (2011). The Transcriptome of the Reference Potato Genome Solanum tuberosum Group Phureja Clone DM1-3 516R44. PLoS ONE 6: e26801.

Matas, A.J. et al. (2011). Tissue- and Cell-Type Specific Transcriptome Profiling of Expanding Tomato Fruit Provides Insights into Metabolic and Regulatory Specialization and Cuticle Formation[W][OA]. Plant Cell 23: 3893-3910. 
Musungu, B., Bhatnagar, D., Brown, R.L., Fakhoury, A.M., and Geisler, M. (2015). A predicted protein interactome identifies conserved global networks and disease resistance subnetworks in maize. Front. Genet. 6: 201.

Myburg, A.A. et al. (2014). The genome of Eucalyptus grandis. Nature.

Naithani, S. et al. (2019). Plant Reactome: a knowledgebase and resource for comparative pathway analysis. Nucleic Acids Res.: gkz996.

Narsai, R., Law, S.R., Carrie, C., Xu, L., and Whelan, J. (2011). In-Depth Temporal Transcriptome Profiling Reveals a Crucial Developmental Switch with Roles for RNA Processing and Organelle Metabolism That Are Essential for Germination in Arabidopsis. Plant Physiol. 157: 1342-1362.

Opitz, N., Marcon, C., Paschold, A., Malik, W.A., Lithio, A., Brandt, R., Piepho, H.-P., Nettleton, D., and Hochholdinger, F. (2016). Extensive tissue-specific transcriptomic plasticity in maize primary roots upon water deficit. J. Exp. Bot. 67: 1095-1107.

Opitz, N., Paschold, A., Marcon, C., Malik, W.A., Lanz, C., Piepho, H.-P., and Hochholdinger, F. (2014). Transcriptomic complexity in young maize primary roots in response to low water potentials. BMC Genomics 15: 741.

Patel, R.V., Nahal, H.K., Breit, R., and Provart, N.J. (2012). BAR expressolog identification: expression profile similarity ranking of homologous genes in plant species. Plant J. 71: 1038-1050.

Ramírez-González, R.H. et al. (2018). The transcriptional landscape of polyploid wheat. Science 361.

Rich-Griffin, C. et al. (2020). Regulation of Cell Type-Specific Immunity Networks in Arabidopsis Roots. Plant Cell 32: 2742-2762.

Righetti, K., Vu, J.L., Pelletier, S., Vu, B.L., Glaab, E., Lalanne, D., Pasha, A., Patel, R.V., Provart, N.J., Verdier, J., Leprince, O., and Buitink, J. (2015). Inference of Longevity-Related Genes from a Robust Coexpression Network of Seed Maturation Identifies Regulators Linking Seed Storability to Biotic Defense-Related Pathways. Plant Cell 27: 2692-2708.

Ryu, K.H., Huang, L., Kang, H.M., and Schiefelbein, J. (2019). Single-Cell RNA Sequencing Resolves Molecular Relationships Among Individual Plant Cells. Plant Physiol. 179: 1444.

Sekhon, R.S., Lin, H., Childs, K.L., Hansey, C.N., Buell, C.R., de Leon, N., and Kaeppler, S.M. (2011). Genome-wide atlas of transcription during maize development. Plant J. 66: 553-563.

Severin, A.J. et al. (2010). RNA-Seq Atlas of Glycine max: A guide to the soybean transcriptome. BMC Plant Biol. 10: 160.

Tai, H., Lu, X., Opitz, N., Marcon, C., Paschold, A., Lithio, A., Nettleton, D., and Hochholdinger, F. (2016). Transcriptomic and anatomical complexity of primary, seminal, and crown roots highlight root type-specific functional diversity in maize (Zea mays L.). J. Exp. Bot. 67: 11231135. 
Tello-Ruiz, M.K. et al. (2018). Gramene 2018: unifying comparative genomics and pathway resources for plant research. Nucleic Acids Res. 46: D1181-D1189.

The Potato Genome Sequencing Consortium (2011). Genome sequence and analysis of the tuber crop potato. Nature 475: 189-195.

The Tomato Genome Consortium (2012). The tomato genome sequence provides insights into fleshy fruit evolution. Nature 485: 635-641.

Thiel, J., Koppolu, R., Trautewig,C., Hertig, C., Kale, S.M., Erbe, S., Mascher, M., Himmelbach, A., Rutten, T., Esteban, E., Pasha, A., Kumlehn, J., Provart, N.J., Vanderauwera, S., Frohberg, C., Schnurbusch, T. (2021). Transcriptional landscapes of floral meristems in barley. Science Advances. DOI: 10.1126/sciadv.abf0832

Tian, C. et al. (2014). An organ boundary-enriched gene regulatory network uncovers regulatory hierarchies underlying axillary meristem initiation. Mol. Syst. Biol. 10: 755.

Toal, T.W. et al. (2018). Regulation of Root Angle and Gravitropism. G3 GenesGenomesGenetics 8: 3841-3855.

Tuskan, G.A. et al. (2006). The Genome of Black Cottonwood, Populus trichocarpa (Torr. \& Gray). Science 313: 1596-1604.

Van Bakel, H., Stout, J.M., Cote, A.G., Tallon, C.M., Sharpe, A.G., Hughes, T.R., and Page, J.E. (2011). The draft genome and transcriptome of Cannabis sativa. Genome Biol. 12: R102.

Van Weringh, A., Pasha, A., Esteban, E., Gamueda, P.J., and Provart, N.J. (2021). Generation of guard cell RNA-seq transcriptomes during progressive drought and recovery using an adapted INTACT protocol for Arabidopsis thaliana shoot tissue. bioRxiv: 2021.04.15.439991.

Waese, J. et al. (2017). ePlant: Visualizing and Exploring Multiple Levels of Data for Hypothesis Generation in Plant Biology. Plant Cell 29: 1806-1821.

Wang, L. et al. (2014). Comparative analyses of C4 and C3 photosynthesis in developing leaves of maize and rice. Nat. Biotechnol. 32: 1158-1165.

Wilkins, O., Nahal, H., Foong, J., Provart, N.J., and Campbell, M.M. (2009a). Expansion and diversification of the Populus R2R3-MYB family of transcription factors. Plant Physiol. 149: 981993.

Wilkins, O., Waldron, L., Nahal, H., Provart, N.J., and Campbell, M.M. (2009b). Genotype and time of day shape the Populus drought response. Plant J. 60: 703-715.

Zhou, R., Macaya-Sanz, D., Rodgers-Melnick, E., Carlson, C.H., Gouker, F.E., Evans, L.M., Schmutz, J., Jenkins, J.W., Yan, J., Tuskan, G.A., Smart, L.B., and DiFazio, S.P. (2018). Characterization of a large sex determination region in Salix purpurea L. (Salicaceae). Mol. Genet. Genomics MGG 293: 1437-1452. 